\title{
Remarks on a paper by Skornyakov concerning rings for which every module is a direct sum of left ideals
}

\author{
By
}

Søren Jøndrop and Claus Michael Ringel.

In [5], Skornyakov claims (as his main result) that a ring $R$ is quasi-Frobenius and serial ("generalised uniserial") if and only if every left $R$-module is a direct sum of left ideals. However, the proof of lemma 4 is incorrect, and there are counterexamples to the theorem itself.

1. Examples. Let $k$ be a field, and $R$ the $k$-algebra given by the quiver (see [3])

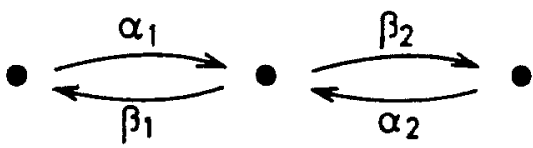

with relations $\alpha_{1} \beta_{2}=\alpha_{2} \beta_{1}=0, \beta_{1} \alpha_{1}=\beta_{2} \alpha_{2}$. Then $R$ is quasi-Frobenius, $(\operatorname{rad} R)^{3}=0$. The separated quiver of $R /(\operatorname{rad} R)^{2}$ is the disjoint union of two quivers of type $A_{3}$, namely $\bullet \rightarrow \bullet \bullet$ and $\bullet \leftarrow \bullet \rightarrow \bullet$, thus any indecomposable module with socle length 2 has multiplicityfree socle (i.e. the composition factors occur with multiplicity $\leqq 1$ in the socle). Of course, the same is true for the remaining six indecomposable modules. This shows that any indecomposable left module can be embedded into the injective cogenerator ${ }_{R} R$. As a consequence, every left module is the direct sum of left ideals, but $R$ is not serial. - If we consider the $k$-algebra $S$ given by the quiver

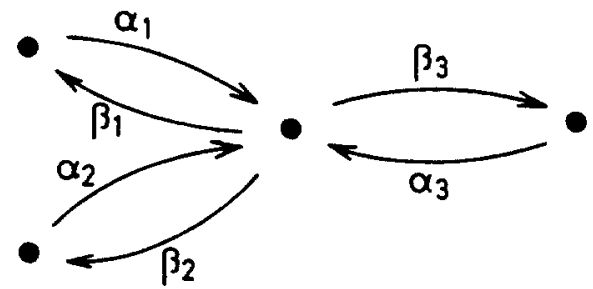

with relations $\alpha_{i} \beta_{j}=0$, for $i \neq j$, and $\beta_{1} \alpha_{1}=\beta_{2} \alpha_{2}=\beta_{3} \alpha_{3}$, then we obtain an example of a ring $S$ with two indecomposable left $S$-modules which are not left ideals, such that, however, every left $M_{2}(S)$-module is the direct sum of left ideals. (Here, $M_{n}(R)$ denotes the ring of all $n \times n$-matrices over $R$ ). 
2. In general. Let $R$ be a quasi-Frobenius ring of finite representation type. Let $S_{1}, \ldots, S_{t}$ be the simple left $R$-modules, $E_{1}, \ldots, E_{t}$ their injective hulls. Since ${ }_{R} R$ is injective, ${ }_{R} R=\bigoplus_{i} E_{i}^{r_{i}}$, with $r_{i} \in \mathbb{N}$, and, since ${ }_{R} R$ is a cogenerator, all $r_{i}>0$. If $M$ is a left module with socle $\oplus S_{i}^{m_{4}}$, then $M$ is embeddable into ${ }_{R} R$ if and only if $m_{i} \leqq r_{i}$, for all $i$. Now let $M_{1}, \ldots, M_{q}$ be the indecomposable left $R$-modules. It has been shown in [4] that all $M_{i}$ are of finite length and that every left $R$-module is a direct sum of copies of these modules. Let $\oplus S_{i}^{m_{i j}}$ be the socle of $M_{j}$, and define $s_{i}=\max _{j} m_{i j}$. It is now clear that every left $R$-module is a direct sum of left ideals if and only if $s_{i} \leqq r_{i}$ for all $i$. If we replace $R$ by the matrix $\operatorname{ring} M_{n}(R)$, then the numbers $r_{i}$ are replaced by $n r_{i}$, whereas the numbers $s_{i}$ are not changed at all. Thus, we see: given a quasi-Frobenius ring $R$ of finite representation type, there exists $n_{0} \in \mathbb{N}$ such that for any $n \geqq n_{0}$, every left $M_{n}(R)$-module is a direct sum of left ideals.

3. Conversely. Assume every left $R$-module is a direct sum of left ideals. Then every injective left $R$-module being embeddable into a free module, has to be projective, thus by a theorem of Faith-Walker [2], $R$ is quasi-Frobenius. Also, the length of the indecomposable left $R$-modules of finite length is bounded by the length of ${ }_{R} R$, thus by a theorem of Auslander [1], $R$ is of finjte representation type.

4. We have seen that the property considered by Skornyakov is not even invariant under Morita equivalence. We note however, that the property is left-right symmetric, and coincides with the property of being a quasi-Frobenius Köthe ring:

Theorem. Let $R$ be quasi-Frobenius. Then the following properties are equivalent:

(1) Every left module is a direct sum of left ideals.

(2) Every left module is a direct sum of cyclic modules.

(1*) Every right module is a direct sum of right ideals.

(2*) Every right module is a direct sum of cyclic modules.

Proof. (1) $\Rightarrow(2)$. Let $M$ be a left ideal which we may assume to be indecomposable. If $M$ is projective, then $M$ is cyclic. If $M$ is not projective, let $\varepsilon: P \rightarrow M$ be a minimal projective cover, and $K$ the kernel of $\varepsilon$. Then it is well-known that $K$ is indecomposable and $P$ its injective hull. Since by assumption $K$ is embeddable into ${ }_{R} R$, we see that $P$ is a direct summand of ${ }_{R} R$, thus $M$ is cyclic.

(2) $\Rightarrow\left(1^{*}\right)$. Consider the duality $D=\operatorname{Hom}_{R}\left(-,{ }_{R} R\right)$ from left $R$-modules to right $R$-modules. Any indecomposable right module of finite length is of the form $D\left({ }_{R} M\right)$ for some indecomposable left module ${ }_{R} M$. Now, by assumption, there is a surjective $\operatorname{map}_{R} R \rightarrow{ }_{R} M$, thus we obtain an injective $\operatorname{map} D\left({ }_{R} M\right) \rightarrow D\left({ }_{R} R\right)=R_{R}$. 


\section{References}

[1] M. Adslander, Representation theory of Artin Algebras II. Communications in Algebra 1, 293-310 (1974).

[2] C. FAITH and E. Walker, Direct-sum representations of injective modules. J. Algebra is, 203-221 (1967).

[3] P. GABRIEL, Unzerlegbare Darstellungen. Manuscripta Math. 6, 71-103 (1972).

[4] C. M. RingeL and H. TACHIKAWA, $Q F-3$ rings. J. reine angew. Math. 272, 49-72 (1975).

[5] L. A. Skonnyakov, Decomposition of modules into a direct sum of ideals. Math. Notes (Translation of Matematicheskie Zametki) 20, 665-668 (1976).

Eingegangen am 18.9.1978

Anschrift der Autoren:

S. Jøndrup

Kobenhavns Universitets Matematigke Institut

Universitetsparken 5

2100 Kobenhavn ø, Danmark
C. M. Ringel

Fakultät für Mathemalik

Universität

D.4800 Bielefeld 